\title{
Filtering Inversion Analysis of Elastic Parameter of U-Shaped Beam
}

\author{
Wang Feng ${ }^{1,}$, Liang Qingyu ${ }^{2, b}$ and Zhang Jian ${ }^{3, c}$ \\ 1 Jiangsu Yangtze River Bridge Co., Ltd., Jiangsu Nanjing China \\ ${ }^{2}$ Zhejiang Institute of Hydraulics and Estuary, Zhejiang Hangzhou China \\ ${ }^{3}$ Nanjing University of Aeronautics and Astronautics, Jiangsu Nanjing China \\ a wfnj1976@126.com, bliangqy@126.com, zjmech@163.com
}

\begin{abstract}
Keywords: Stochastic filtering, inversion analysis, U-shaped beam, elastic parameter
Abstract. With the development of civil engineering, the U-shaped beam has been widely applied in practical engineering. In general, it is composed of concrete and steel and compared with mechanical analysis, little research has been carried on the inversion analysis of elastic parameter of U-shaped beam. With degraded solid element theory, the shell element is deduced and the displacement function is obtained. The necessary observation revision equation and Kalman regenerative matrix are derived. The stochastic filtering inversion analysis steps of elastic parameter of the U-shaped beam are presented and the analytical procedure is compiled. Through analysis of a classic example, some important conclusions about stochastic filtering inversion analysis of elastic parameter of U-shaped beam are obtained.
\end{abstract}

\section{Introduction}

According to the characteristics of highway bridges and requirements, the new method to design and calculation method of highway concrete channel beam is used, and not is completely copying the design and calculation method of existing railway channel beam. Concrete beam is in the form of I-shaped, $\Gamma$-shape, U-shape and box, as shown. If the span is not available, we can use the I-shaped; if not, we can use the box; In order to reduce edge beam spacing, that is reducing the transverse span of driving but, concrete beam can turn into inclined edge beam, and we can use $\Gamma$-shape, U-shaped channel beam is more used in building the girders of rail transit in our country in recent years. In the past few decades, no more attention has been attracted to the determination of mechanical parameters such as elastic parameter of the engineering structure than to the mechanical analysis [1-3]. Fortunately in recent years, more and more scholars have realized the importance of the determination of mechanical parameters of the engineering structure. Accordingly the research emphasis has been transferred to it and many research results have been gained [4-6]. The U-shaped beam has been widely applied in practical engineering. With the development of civil engineering, the U-shaped beam is more and more widely used. For a long time, emphasis has been put on mechanical analysis of the U-shaped beam. Some computational codes besides general FEM software have solved it well.

Thus, the present study is to carry out the stochastic filtering of elastic parameter of the U-shaped beam in detail.

\section{Stochastic filtering theory}

In this paper, the stochastic filtering theory is referred to the extended Kalman filtering theory. The filtering practical equations for U-shaped beam are derived and achieved as follows:

$$
\begin{aligned}
& \mathbf{E}_{i+1}=\mathbf{E}_{i+1, i}+\mathbf{K}_{i+1}\left[\mathbf{U}_{i+1}^{*}-\mathbf{U}_{i+1}\left(\mathbf{E}_{i+1, i}\right)\right] \\
& \mathbf{P}_{i+1}=\mathbf{P}_{i+1, i}-\mathbf{K}_{i+1} \mathbf{H}\left(\mathbf{E}_{i+1, i}\right) \mathbf{P}_{i+1, i}
\end{aligned}
$$

Where Eqs.(1-2) are the observation revision equations. $\mathbf{E}_{i}$ and $\mathbf{E}_{i+1, i}$ are respectively the $i$ th

filtering value and the $i$ th predicting value of elastic parameter $\mathbf{E} . \mathbf{U}_{i}$ and $\mathbf{U}_{i}^{*}$ are respectively the $i$ th calculative displacement vector and the $i$ th measured displacement vector. $\mathbf{P}_{i}$ is the $i$ th 
estimating error covariance matrix. $\mathbf{P}_{i+1, i}$ is the $i$ th predicting error covariance matrix. $\mathbf{K}_{i}$ is the $i$ th Kalman regenerative matrix of great importance, which is expressed as:

$$
\mathbf{K}_{i+1}=\mathbf{P}_{i+1, i} \mathbf{H}^{T}\left(\mathbf{E}_{i+1, i}\right)\left[\mathbf{H}\left(\mathbf{E}_{i+1, i}\right) \mathbf{P}_{i+1, i} \mathbf{H}^{T}\left(\mathbf{E}_{i+1, i}\right)+\mathbf{R}_{i+1}\right]^{-1}
$$

Where $\mathbf{R}_{i}$ is the measurement error covariance matrix of the thin-walled multi-cell box girder structure, which can be omitted or supposed to a very small value. And the sensitivity matrix $\mathbf{H}\left(\mathbf{E}_{i+1, i}\right)$ can be calculated with finite difference method. The rest equations are listed as:

$$
\begin{aligned}
& \mathbf{E}_{i+1, i}=\boldsymbol{\Phi}_{i+1, i} \mathbf{E}_{i} \\
& \mathbf{P}_{i+1, i}=\boldsymbol{\Phi}_{i+1, i} \mathbf{P}_{i} \boldsymbol{\Phi}_{i+1, i}^{T}+\mathbf{Q}_{i+1}
\end{aligned}
$$

Where $\mathbf{Q}_{i}$ is the systematic error covariance matrix of the studied thin-walled multi-cell box girder structure. $\boldsymbol{\Phi}_{i+1, i}$ is the $i$ th status transferring matrix, which can be defined as unit matrix $\mathbf{I}$ in extended Kalman filtering identification analysis. In fact, Eqs.(5-6) are unnecessary and without employment in this paper. And for iterative problem, the significant initial conditions must be considered as:

$$
\mathbf{E}_{0}=\hat{\mathbf{E}}_{0}, \quad \mathbf{P}_{0}=\hat{\mathbf{P}}_{0}
$$

Where $\mathbf{E}_{0}$ is the initial value of elastic parameter $\mathbf{E}$, which can be supposed by practical engineering experiences instead of arbitrary selection. And $\mathbf{P}_{0}$ is the initial error covariance matrix, which can be supposed to a large value generally and if the Kalman iterative processes are convergent to the true value, $\mathbf{P}_{0}$ will be descendent to a small value. Obviously, $\mathbf{U}_{i}, \mathbf{P}_{i}$ and $\mathbf{K}_{i}$ are all the functions of elastic parameter $\mathbf{E}$, which are determined with combination of finite strip element method for the thin-walled multi-cell box girder structure.

\section{Layered shell element}

The continuous U-shaped channel beam consist of concrete, Common reinforced and prestressed reinforcement, the layer unit cannot accurately simulate the geometrical configuration of prestressed reinforcement, so it is simulated by the composite shell element(as shown in Fig.1), the node displacement array of degenerate shell element is:

$$
\delta_{i}=\left[\begin{array}{lllll}
u_{i} & v_{i} & w_{i} & \beta_{1 i} & \beta_{2 i}
\end{array}\right]^{\mathrm{T}}
$$

In this formula: $\left[\begin{array}{lll}u_{i} & v_{i} & w_{i}\end{array}\right]$ is the line displacement of node $\mathrm{i}$ in global coordinate system; $\left[\begin{array}{ll}\beta_{1 i} & \beta_{2 i}\end{array}\right]$ is the angle displacement of node $\mathrm{i}$ in node coordinate system.
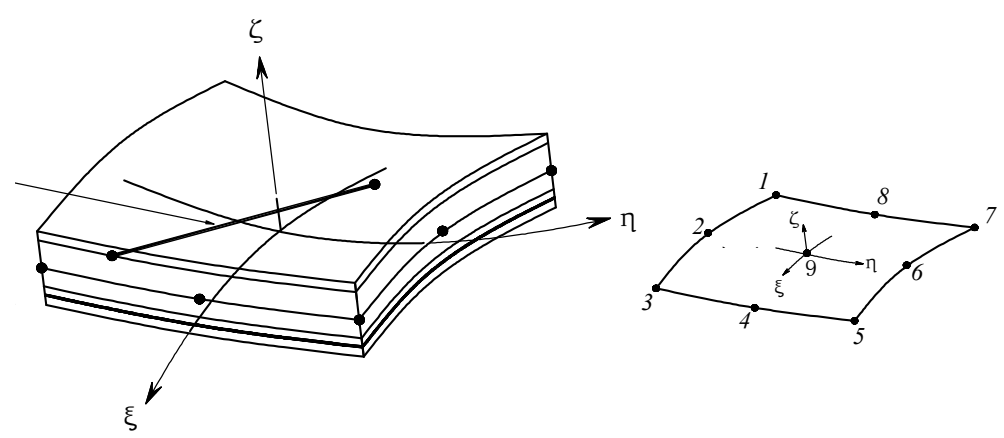

Fig. 1 Element model

Through the shape function interpolating, the displacement field shows as follows:

$$
\begin{gathered}
u=\sum_{i=1}^{n} N_{i} u_{i}+\sum_{i=1}^{n} N_{i} \frac{h_{i}}{2} \zeta\left(v_{1 i}^{x} \beta_{1 i}-v_{2 i}^{x} \beta_{2 i}\right) \\
v=\sum_{i=1}^{n} N_{i} v_{i}+\sum_{i=1}^{n} N_{i} \frac{h_{i}}{2} \zeta\left(v_{1 i}^{y} \beta_{1 i}-v_{2 i}^{y} \beta_{2 i}\right)
\end{gathered}
$$




$$
w=\sum_{i=1}^{n} N_{i} w_{i}+\sum_{i=1}^{n} N_{i} \frac{h_{i}}{2} \zeta\left(v_{1 i}^{z} \beta_{1 i}-v_{2 i}^{z} \beta_{2 i}\right)
$$

In this formula: $u 、 v 、 w$ is the displacement field of degenerate shell element; $n$ is the sum of node about degenerate shell element; $N_{i}$ is the shape function of node I; $h_{i}$ is the shell element thickness of node $\mathrm{I} ; v_{1 i}^{x}$ is the arccosine of node coordinate system $v_{1}$ to the $\mathrm{x}$ axis in the global coordinate system,so is $v_{1 i}^{y}$ to $\mathrm{y}$ axis and $v_{1 i}^{z}$ to $\mathrm{z}$ axis; $v_{2 i}^{x}$ is the arccosine of node coordinate system $v_{2}$ to the $\mathrm{x}$ axis in the global coordinate system,so is $v_{2 i}^{y}$ to y axis and $v_{2 i}^{z}$ to $\mathrm{z}$ axis.

\section{Steps of stochastic filtering inversion analysis}

(1) The measured displacement data $\mathbf{U}_{1}^{*}, \mathbf{U}_{2}^{*}, \cdots, \mathbf{U}_{n}^{*}$ of the U-shaped beam structure are gathered. $\mathbf{U}_{k}^{*}(k=1, \cdots, n)$ is the measured displacement vector of the $k$ th time of the measured displacements;

(2) Suppose the elastic parameter vector $\boldsymbol{E}$. Set the initial value $\mathbf{E}_{0}$ with practical engineering experiences the main element of $\mathbf{P}_{0}$ is supposed to a large value; set $\varepsilon$ as the filtering convergence criterion and $i$ as filtering time variable;

(3) Input the $i$ th measured displacement vector $\mathbf{U}_{i}^{*}$ and determine the $i$ th calculative displacement vector $\mathbf{U}_{i}$ of the $U$-shaped beam structure with finite strip element method; input the measurement error covariance matrix $\mathbf{R}_{i}$, which can be omitted or supposed to a very small value;

(4) Compute the residual displacement vector $\mathbf{U}_{i}^{*}-\mathbf{U}_{i}$ of the $U$-shaped beam structure and the matrix $\mathbf{H}\left(\mathbf{E}_{i+1, i}\right)$ by finite difference method; calculate the Kalman plus matrix $\mathbf{K}_{i}$ and then determined the residual plus matrix $\Delta \mathbf{E}_{i}=\mathbf{K}_{i}\left(\mathbf{U}_{i}^{*}-\mathbf{U}_{i}\right)$;

(5) Combined with Eq.(11), the $i$ th filtering identification value $\mathbf{E}_{i}$ of the elastic parameter $\mathbf{E}$ of the U-shaped structure is worked out. And then determine the $i$ th convergence parameter $\beta_{i}=\max _{1 \leq j \leq 4}\left(\frac{\Delta E_{j}}{E_{j}}\right)_{i}$;

(6) If $\beta_{i}<\varepsilon$, the filtering iterative processes are convergent and the final identification result $\mathbf{E}_{i}$ of the elastic parameter $\mathbf{E}$ of the U-shaped beam structure is obtained. Otherwise, set the filtering time variable $i=i+1$ and then return to step (3) to continue filtering inversion analysis.

\section{Analysis of an example}

The U-shaped beam is shown in Fig. 2. The total length of the studied U-shaped beam is is referred to Ref.[8]. The elastic parameter of the top board is written as $E_{t}$ and that of the abdomen board is $E_{a}$. The elastic parameter vector $\mathbf{E}$ are $\left[E_{t}, E_{a}\right]$, which are both equal to $100 \mathrm{GPa}$. In order to carry on Kalman filtering inversion analysis of elastic parameter, the corresponding filtering procedure is developed. The U-shaped beam is modeled by layered shell element shown in Fig. 2.

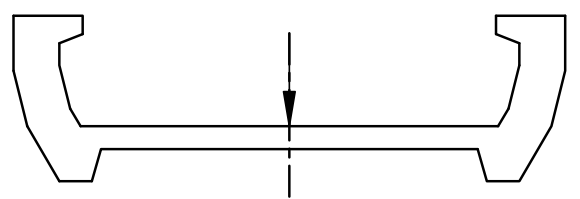

Fig. 2. Cross sections of composite T-beam 
Respectively select the initial values $\boldsymbol{E}_{1}=[200,200]$ and $\boldsymbol{E}_{2}=[50,50]$, which are put into the stochastic filtering inversion analysis procedure together with the data in Table 1. Suppose the convergence criteria $\varepsilon=0.1$ and the iterative results of elastic parameter are listed in Table 1 .

Table 1. Results of stochastic filtering inversion analysis of the U-shaped beam /GPa

\begin{tabular}{ccccc}
\hline Mechanical & $E_{t}$ & $E_{a}$ & $E_{t}$ & $E_{a}$ \\
Parameters & 200 & 200 & 50 & 50 \\
\hline Initial value & 100.24 & 100.31 & 99.27 & 99.30 \\
Final value & 14 & 14 & 22 & 22 \\
Iterative times & 14 & & 22 \\
\hline
\end{tabular}

Evidently, differences between the two groups of results are very small. The average parameter of the top board and the abdomen board are respectively100.24GPa and 100.31GPa. Elastic parameter of the top board and the abdomen board of the U-shaped beam are all viewed as random variables. The results demonstrate that the adopted stochastic filtering theory is feasible and reliable for the identification analysis and the compiled analytical procedure are correct and authentic.

\section{Conclusion}

The filtering process of stochastic inversion analysis of elastic parameter of the U-shaped beam is steadily convergent to the true values. The adopted stochastic filtering inversion analysis is correct and authentic. And the complicated error function and the optimization method are utilized in the stochastic filtering inversion analysis, which proves the simplicity and practicability of the filtering method.

\section{Acknowledgments}

This work was financially supported by the National Natural Science Foundation of China (Nos. 11232007 and 11272147), Natural Science Foundation of Jiangsu Province(BK20130787), State Key Laboratory of Mechanics and Control of Mechanical Structures(MCMS-0213G01), Hydraulics Science Project of Zhejiang Province(RC1445), Youth Science and Technology Innovation Fund of NUAA(NS2014003), Science Project of Jiangsu Yangtze River Bridge Co., Ltd.( GCJS2014-37).

\section{References}

[1] A. G. Razaqpur, and M. Nofal: Can. J. Civil Eng. Vol. 11 (1989) , p. 459

[2] S. V. Modak, T. K. Kundra, and B. C. Nakra: J. Sound Vib. Vol. 254 (2002) , p. 447

[3] S. Y. Chi, J. C. Chern, and C. C. Lin: Tun. Und. Space Technol. Vol. 16 (2001) , p. 159

[4] M. Sanayei, and M. J. Saletnik: J. Struct. Eng., Vol. 122 (1996) , p. 555

[5] J. Zhang, J. S. Ye, and X. X. Shi: J. Nanjing Univ. Aero. Astro. Vol. 42 (2010), p. 262

[6] E. Radin, and L. Vahid: Compos. Struct. Vol. 81 (2003), p. 1461

[7] Z. Fang, C. Gong, J. Yang, and T. I. Campbell: J. Cent. South Univ. Technol. Vol. 16 (2009), p. 495

[8] J. Zhang, W. G. Lan, and B. Yu: Adv. Mater. Res. Vol. 163 (2011), p. 1874

[9] A. G. Razaqpur, and M. Nofal: Can. J. Civil Eng. Vol. 11(1989), p. 459

[10]J. Zhang, W. G. Lan, and J. Lin: Adv. Mater. Res. Vol. 146 (2011), p. 1519

[11] S. Y. Chi, J. C. Chern, and C. C. Lin: Tun. Und. Space Technol. Vol. 16 (2001), p. 159

[12] J. Zhang, C.W. Zhou, W. G. Lan, and et al: Chinese J. Appl. Mech. Vol. 27 (2010), p. 746 